\title{
ROTATIONAL PERTUBATION OF MASIVE SCALAR FIELD UNIVERSES
}

\section{NAOREM JUGESHWOR SINGH}

\author{
THAMBAL MARIK COLLEGE,OINAM DEPARTMENT OF MATHEMATICS P/O,P/S : NAMBOL, \\ MANIPUR - 795134
}

Keywords: Massive scalar, rotational and non rotational universe, Astrophysics object.

\begin{abstract}
In this course of presentation, we will consider the rotational perturbation of the Robertson-Walker universe will be examined in order to substantiate the possibility of the existence of Massive scalar field. We will study, the exact solutions for metric rotation $\Omega(\mathrm{r}, \mathrm{t})$ and the matter rotation $\mathrm{w}(\mathrm{r}, \mathrm{t})$ under different conditions, and also study their nature and role from different angles.
\end{abstract}

\section{INTRODUCTION}

The studies of rotating astrophysical bodies coupled with gravitational field in presence of other fields are so far done by Bayin ( 1981,1985) ; Krori it al. ( 1983); Van den Bergh and Wils (1984); Islam ( 1985); Tiwari it al ( 1986) and Koijam ( 1987, 1988). Many authors have obtained models of rotating objects without expansion and expanding object without rotation. Thus it will be of great interest to find out explicitly solved models of expanding as well as rotating objects so that information's about the behavior of the universe can be obtained from such models. Thus here we investigate rotating as well as expanding models.

The Robertson-Walker models are believed to be appropriate for a representation of large scale structure of the space-time, we consider this type of metric here for our problem. Furthermore, as object of our study, we take up rotational perturbation of Massive scalar field as it will be very stimulating to make investigations on such models in trying to obtain new information's concerning rotating astrophysical objects in this universe and we can draw many conclusions for a realistic universe from such studies. In many respects our problem will be very interesting as, through in most of the Robertson Walker metric can be used for general relativity solutions with rotating and massive scalar field, and many stimulating findings for further research may be obtained from it. The study of the rotational perturbations of these models are also made in order to substantiate the possibility that the universe is endowed with slight rotation in the course of presentation of several analytic solutions.

\section{FIELD EQUATION}

The metric considered here is the perturbation from of the Robertson-Walker metric viz.

$$
\mathrm{ds}^{2}=\mathrm{dt}^{2}-\mathrm{R}(\mathrm{t})\left[\frac{d r 2}{1-k r 2}+r 2 d \Theta^{2}+r^{2} \operatorname{Sin} \Theta d \phi^{2}\right]+2 \Omega(\mathrm{r}, \mathrm{t}) \mathrm{R}^{2}(\mathrm{t}) \mathrm{r}^{2} \sin ^{2} \Theta \mathrm{d} \phi \mathrm{dt},
$$

where $\Omega(\mathrm{r}, \mathrm{t})$ is the metric rotation function which is related to the local dragging of the inertial forms.

The energy momentum tensor taken up for this problem is that of the massive scalar field given by

$$
\mathrm{T}_{i j}=\frac{1}{4 \pi}\left[\phi_{i} \phi_{j}-\frac{1}{2} \mathrm{~g}_{i j}\left(\phi_{i} \phi^{i}-\mathrm{M}^{2} \phi\right)\right] \text {, }
$$

where the scalar potential $\phi$ satisfied the Klein- Gordon equation. 


$$
\mathrm{g}^{i j} \phi ; i j+\mathrm{M}^{2} \phi=\epsilon
$$

Here $\in$ is the source density of the scalar field and $M$ is related to the mass of the spin particle by.

$$
\mathrm{M}=m / \hbar \quad \text { where } \hbar=\frac{h}{2 \pi}
$$

( $\mathrm{h}$ being the Plank's constant)

Here the spatial velocity distribution is given by

$$
\mathrm{v}^{i}=\frac{d x^{i}}{d t}=\left(0,0, v^{3}\right)
$$

and in this case

$$
\mathrm{u}_{\propto}=(0,0,-\mathrm{g} 33, \Omega, 1)
$$

Now considering terms up to the first order in $\Omega$, the Einstein field equation gives

$$
\begin{aligned}
& 2 \frac{\stackrel{\mathrm{R}}{\mathrm{R}}}{\mathrm{R}^{2}}+\frac{\mathrm{R}^{2}}{\mathrm{R}^{2}}+\mathrm{k}\left[\frac{\mathrm{M}^{2}}{\mathrm{R}^{2}}-\dot{\varphi}^{2}-\left(\frac{1-\mathrm{kr}^{2}}{\mathrm{R}^{2}}\right) \varphi^{\prime 2}\right], \\
& 2 \frac{\stackrel{\mathrm{R}}{\mathrm{R}}}{\mathrm{R}^{2}}+\frac{\mathrm{R}^{2}}{\mathrm{R}^{2}}=\mathrm{G}\left[\frac{\mathrm{M}^{2}}{\mathrm{R}^{2}}-\dot{\varphi}^{2}+\left(\frac{1-\mathrm{kr}^{2}}{\mathrm{R}^{2}}\right) \varphi^{\prime 2}\right], \\
& 3\left(\frac{\mathrm{R}^{2}}{\mathrm{R}^{2}}+\frac{\mathrm{k}}{\mathrm{R}^{2}}\right)=\mathrm{G}\left[\dot{\varphi}^{2}+\frac{\mathrm{M}^{2}}{\mathrm{R}^{2}}+\left(\frac{1-\mathrm{kr}^{2}}{\mathrm{R}^{2}}\right) \varphi^{\prime 2}\right], \\
& 3 \frac{\stackrel{\circ}{\mathrm{R}}}{\mathrm{R}} \Omega^{\prime}+\stackrel{\circ}{\Omega^{\prime}}=0, \\
& \left(\mathrm{R} \stackrel{\circ}{\mathrm{R}}+2 \mathrm{R}^{2}+2 \mathrm{k}\right) \Omega=\frac{1}{2}\left(1-\mathrm{kr}^{2}\right) \Omega^{\prime /}+\frac{2}{\mathrm{r}}\left(1-\frac{5}{4} \mathrm{kr}^{2}\right) \Omega^{\prime},
\end{aligned}
$$

From Equation (5) and (6), we have

$$
\varphi^{\prime}=0 \text {, }
$$

Again Equations (6) and (7) gives with the help of relation (10) gives

$$
\begin{aligned}
& \frac{\mathrm{R}}{\mathrm{R}}+2 \frac{\mathrm{R}^{2}}{\mathrm{R}^{2}}+\frac{2 \mathrm{k}}{\mathrm{R}^{2}}=\mathrm{G} \frac{\mathrm{M}^{2}}{\mathrm{R}^{2}} \\
& \text { i.e. } \mathrm{R} \stackrel{\circ}{\mathrm{R}}+2 \mathrm{R}^{2}+2 \mathrm{k}=\mathrm{GM}^{2},
\end{aligned}
$$


Now equation (9) with the help of relation (11) we get

$$
\frac{1}{2}\left(1-\mathrm{kr}^{2}\right) \Omega^{\prime \prime}+\frac{2}{\mathrm{r}}\left(1-\frac{5}{4} \mathrm{kr}^{2}\right) \Omega^{\prime \prime}=\mathrm{GM}^{2} \Omega,
$$

Again Equation (8) gives

$$
\Omega(r, t)=L(r) R^{-3}+N(t),
$$

Now making use of the relation (13) in equation (12), we get

$$
\begin{aligned}
& \frac{1}{2}\left(1-\mathrm{kr}^{2}\right) \mathrm{L}^{\prime \prime}+\frac{2}{\mathrm{r}}\left(1-\frac{5}{4} \mathrm{kr}^{2}\right) \mathrm{L}^{\prime}=\mathrm{GM}^{2} \mathrm{~L} \\
& \text { i.e. }\left(1-\mathrm{kr}^{2}\right) \frac{\mathrm{L}^{\prime \prime}}{\mathrm{L}}+\left(\frac{4}{\mathrm{r}}-5 \mathrm{kr}\right) \frac{\mathrm{L}^{\prime}}{\mathrm{L}}-2 \mathrm{GM}^{2}=0,
\end{aligned}
$$

CASE . I

Now considering $\mathrm{k}=1$ which corresponding to closed models. Here using the substituting $\mathrm{y}=\mathrm{kr}^{2}$ in (14), we get.

$$
\mathrm{y}(1-\mathrm{y}) \mathrm{L}_{\mathrm{yy}}+\left(\frac{5}{2}-3 \mathrm{y}\right) \mathrm{L}_{\mathrm{y}}-\frac{2 \mathrm{GM}^{2}}{4 \mathrm{k}} \mathrm{L}=0,
$$

We see that equation (15) is similar to the hypergeometric equation

$$
\mathrm{y}(1-\mathrm{y}) \mathrm{F}_{\mathrm{yy}}+[\gamma-(1+\alpha+\beta) \mathrm{y}] \mathrm{F}_{\mathrm{y}}-\alpha \beta \mathrm{F}=0,
$$

of which the general solution is given by

$$
\mathrm{F}=\mathrm{A}_{0} \mathrm{~F}(\alpha, \beta ; \mathrm{v} ; \mathrm{v})+\mathrm{A}_{1} \mathrm{y}^{1-\mathrm{v}} \mathrm{F}(1-\mathrm{v}+\alpha, 1-\mathrm{v}+\beta, \mathrm{z}-\mathrm{v} ; \mathrm{y}),
$$

where $\mathrm{A}_{0}$ and $\mathrm{A}_{1}$ are arbitrary constants and

$$
\mathrm{F}(\alpha, \beta ; v ; \mathrm{y})=\sum_{\mathrm{n}=0}^{\infty} \frac{(\alpha)_{\mathrm{n}}(\beta)_{\mathrm{n}}}{\mathrm{n} !(v)_{\mathrm{n}}} \mathrm{y}^{\mathrm{n}},
$$

Thus in this case we get the general solution of equation (15) as

$$
L(r)=A_{0} \sum_{n=0}^{\infty} \frac{(\alpha)_{n}(\beta)_{n}}{n !\left(\frac{5}{2}\right)_{n}} y^{n}+A_{1} y^{-3 / 2} \sum_{n=0}^{\infty} \frac{\left(\alpha-\frac{3}{2}\right)_{n}\left(\beta-\frac{3}{2}\right)_{n}}{n !\left(-\frac{1}{2}\right)_{n}} y^{n},
$$

since the second term is not regular at $\mathrm{y}=0$

we take $\mathrm{A}_{1}=0$, then we get

$$
L(r)=A_{0} \sum_{n=0}^{\infty} \frac{(\alpha)_{n}(\beta)_{n}}{n !\left(\frac{5}{2}\right)_{n}} y^{n}
$$




$$
\text { i.e. } L(r)=A_{0}(1-y)^{\frac{5}{2}-\alpha-\beta} \sum_{n=0}^{\infty} \frac{\left(\frac{5}{2}-\alpha\right)_{n}\left(\frac{5}{2}-\beta\right)_{n}}{n !\left(\frac{5}{2}\right)_{n}} y^{n} \text {, }
$$

Now we give some of the explicit solutions :

(i) If

$$
\begin{aligned}
& \alpha=-\frac{1}{2}, \beta=\frac{5}{2}, \text { then } \\
& F\left(-\frac{1}{2}, \frac{5}{2} ; 5 ; y\right)=(1-y)^{\frac{1}{2}}
\end{aligned}
$$

(ii) If $\alpha=\frac{9}{2}, \beta=-\frac{5}{2}$ then we have

$$
\mathrm{F}\left(\frac{9}{2},-\frac{5}{2} ; \frac{5}{2} ; \mathrm{y}\right)=(1-\mathrm{y})^{\frac{1}{2}}\left(1-4 \mathrm{y}+\frac{24}{7} \mathrm{y}^{2}\right)
$$

In this case, we get

$$
\Omega(\mathrm{r}, \mathrm{t})=\mathrm{A}_{0}\left(1-\mathrm{kr}^{2}\right)^{\frac{1}{2}}\left(1-4 \mathrm{kr}^{2}+\frac{24}{7} \mathrm{kr}^{2}\right) \mathrm{R}^{-3}+\mathrm{N}(\mathrm{t}),
$$

(iii) If

$$
\mathrm{F}\left(-1,3, \frac{5}{2} ; \mathrm{y}\right)=1-\frac{6}{5} \mathrm{y}
$$

In this case, $\alpha=-1, \beta=3$ and

$$
\Omega(\mathrm{r}, \mathrm{t})=\mathrm{A}_{0}\left(1-\frac{6}{5} \mathrm{kr}^{2}\right) \mathrm{R}^{-3}+\mathrm{N}(\mathrm{t})
$$

(iv) Now

$$
\mathrm{F}\left(4,-2 ; \frac{5}{2} ; \mathrm{y}\right)=\mathrm{A}_{0}\left(1-\frac{16}{5} \mathrm{kr}^{2}+\frac{16}{7} \mathrm{k}^{2} \mathrm{r}^{4}\right)
$$

$$
\Omega(r, t)=A_{0}\left(1-\frac{16}{5} \mathrm{kr}^{2}+\frac{16}{7} \mathrm{k}^{2} \mathrm{r}^{2}\right) \mathrm{R}^{-3}+\mathrm{N}(\mathrm{t}),
$$

In case (1), there, for $z=2 \mathrm{GM}^{2}$, we get

$\mathrm{R}=\left(\mathrm{m}_{0} \mathrm{t}+\mathrm{n}_{0}\right)^{1 / 3}$ 
CASE . 2

In this case, we consider open models which correspond to $\mathrm{k}=-1$. we obtain here different value of $\Omega$ corresponding to different value of $\mathrm{M}$.

If we take $M^{2}=\frac{3}{2 G}$, then from equation (14) we get

$$
L(r)=\frac{C_{0}}{2} r^{-2}\left(1-r^{2}\right)^{\frac{1}{2}}-\frac{C_{0}}{2} r^{-3} \sinh ^{-1} r+C_{1} r^{-3}
$$

where $\mathrm{C}_{0}$ and $\mathrm{C}_{1}$ are arbitrary constants,

Therefore,

$\Omega(r, t)=\left[\frac{C_{0}}{2} r^{-3}\left(1-r^{2}\right)^{\frac{1}{2}}-\frac{C_{0}}{2} r^{-3} \sinh ^{-1} r+C_{1} r^{-3}\right] R^{-3}+N(t)$,

Again if $\mathrm{M}^{2}=5 / \mathrm{G}$, we get

$L(r)=\left\{C_{2}\left(1+r^{2}\right)^{\frac{1}{2}}-\frac{C_{3}}{5}\left(8 r+4 r^{-1}-r^{-3}\right)\right\}$

where $\mathrm{C}_{2}$ and $\mathrm{C}_{3}$ are arbitrary constant.

Therefore,

$\Omega(r, t)=\left[C_{2}\left(1+r^{2}\right)^{\frac{1}{2}}-\frac{C_{3}}{3}\left(8 r+4 r^{-1}-r^{-3}\right)\right] R^{-3}+N(t)$,

If $\mathrm{M}^{2}=-\frac{4}{\mathrm{G}}$, we have

$$
\mathrm{L}(\mathrm{r})=\mathrm{r}^{-3}\left\{\mathrm{C}_{4} \mathrm{r}-\mathrm{C}_{4}\left(1+\mathrm{r}^{2}\right)^{\frac{1}{2}} \sin ^{-1} \mathrm{r}+\mathrm{C}_{5}\left(1+\mathrm{r}^{2}\right)^{\frac{1}{2}}\right\},
$$

Therefore

$$
\Omega(r, t)=\left[r^{-3}\left\{C_{4} r-C_{4}\left(1+r^{2}\right)^{\frac{1}{2}} \sin ^{-1} r+C_{5}\left(1+r^{2}\right)^{\frac{1}{2}}\right\} R^{-3}+N(t)\right],
$$

where $\mathrm{C}_{4}$ and $\mathrm{C}_{5}$ are arbitrary constants.

CASE . 3

In this case we consider the flat model for which $\mathrm{k}=0$ and here equation (14) becomes

$$
\frac{\mathrm{L}^{\prime \prime}}{\mathrm{L}}+\frac{4}{\mathrm{r}} \frac{\mathrm{L}^{\prime}}{\mathrm{L}}=2 \mathrm{GM}^{2}
$$

which gives 


$$
L(r)=r^{-2}\left[x\left\{A_{1} \exp \left(x^{\frac{1}{2}} r\right)+A_{2} \exp \left(-x^{\frac{1}{2}} r\right)\right\}-x^{\frac{1}{2}} r^{-1}\left\{A_{1} \exp \left(x^{\frac{1}{2}} r\right)-A_{2} \exp \left(-x^{\frac{1}{2}} r\right)\right\}\right]
$$

where A1 and A2 are arbitrary constants, and $\mathrm{x}=2$ GM2

In this case

$\Omega(r, t)=r^{-2}\left[x\left\{A_{1} \exp \left(x^{\frac{1}{2}} r\right)+A_{2} \exp \left(-x^{\frac{1}{2}} r\right)\right\}-x^{\frac{1}{2}} r^{-1}\left\{A_{1} \exp \left(x^{\frac{1}{2}} r\right)-A_{2} \exp \left(-x^{\frac{1}{2}} r\right)\right\} R^{-3}+N(t)\right.$

Now if $M=0$, then we get, from equation (29)

$\mathrm{L}(\mathrm{r})=\mathrm{m}_{1}+\mathrm{m}_{1} \mathrm{r}^{-3}$

where $\mathrm{m}_{1}$ and $\mathrm{n}_{1}$ are arbitrary constants .

Thus in this case we have

$$
\Omega(\mathrm{r}, \mathrm{t})=\left(\mathrm{m}_{1}+\mathrm{n}_{1} \mathrm{r}^{-3}\right) \mathrm{R}^{-3}+\mathrm{N}(\mathrm{t}),
$$

which is incidentally corresponding to the case of perfect dragging

Now here $\mathrm{R}(\mathrm{t})$, making use of equation (11); is found to be

$$
\mathrm{R}=\left(\mathrm{m}_{2} \mathrm{t}+\mathrm{n}_{2}\right)^{1 / 3}
$$

where $\mathrm{m}_{2}$ and $\mathrm{n}_{2}$ are arbitrary constants.

Thus here

$$
\Omega(\mathrm{r}, \mathrm{t})=\left(\mathrm{m}_{1}+\mathrm{n}_{1} \mathrm{r}^{-3}\right) \mathrm{R}^{-3}+\mathrm{N}(\mathrm{t}),
$$

where $\mathrm{N}(\mathrm{t})$ is an arbitrary function of time.

\section{CONCLUSION :}

Case I .

In this case, the rotational perturbation decay with the increase of the time for all the models obtained. It is also observed that the smaller the value of $A_{0}$ the smaller are the value of $\Omega(r, t)$ and $\mathrm{w}(\mathrm{r}, \mathrm{t})$ which means that the massive scalar field slow down the rotation. In this case the expanding factor is given by

$$
\theta=m_{0}\left\{\frac{\left(m_{0} t+n_{0}\right)^{-2}}{m_{0} t+n_{0}}\right\}^{\frac{1}{3}}
$$

and since it is positive, our model universes here are expanding ones. Thus, the models are rotating as well as expanding one which may be taken as examples of realistic models.

\section{Case 2.}

In this case, in all the two open models obtained, we find that the rotational perturbations decay as $r$ increases and also with the increase of time if $\mathrm{N}(\mathrm{t})$ is a decreasing function of the time. For the model obtained for $\mathrm{M}^{2}=-4 / \mathrm{G}$ we get the expansion factor as $\theta=3 \mathrm{~m}_{0} \cot \mathrm{h}\left(\mathrm{m}_{0} \mathrm{t}+\mathrm{n}_{0}\right)$ 
Thus we get a rotating as well as expanding model which can be thought of as one of the value of $\Omega(\mathrm{r}, \mathrm{t})$ becomes smaller which shows that the presence the massive scalar field decreases the rotational motion. When $\mathrm{M}^{2}=5 / \mathrm{G}$ the solution is restricted within the range $-1 \leq \mathrm{r} \leq 1$

Case .3.

For all the models obtained in this case the rotational perturbation falls rapidly with the increase of $\mathrm{r}$. In the case of perfect dragging the matter rotation $\mathrm{w}(\mathrm{r}, \mathrm{t})$ and the rotational velocity $\Omega$ $(r, t)$ is independent of the massive scalar field.

\section{References}

[1] Adler. R.J., J. Math. Phys. 15; 727 ( 1974).

[2] Anderson J.L., Relativity- Proceeding of the Relativity conference in the Midwest, ed. By M. Cermeli, S.I Fickler and L. Witten, Plenum press, New York ( 1969).

[3] Banerjee A. and Santosh, N.O, Phys. Rev. D 23; 2111 ( 1981).

[4] Banerjee A and Santosh, N.O, J. Math.Phys 24; 2689 ( 1983 )

[5] Bayin S.S, Phys. Rev D 21; 2433 ( 1980 ).

[6] Bhamra K.S., Aust. J. Phys 27; 541 ( 1974)

[7] Chandra R, Gen. Rel. Grav. 8; 787 ( 1977).

[8] Coley A.A. and Tupper, B.O.J. Astrophys. J. 271; 1 ( 1983)

[9] Coley A.A. and Tupper, B.O.J., Phys. Letters 95A; 357 ( 1983)

[10]Coley A.A. and Tupper, B.O.J., Phys Rev. D., 29; 2701 ( 1984)

[11]Heller. M , Ostrowski, M., Szydlowski, M. and Wosczyne, A. ,Astroph. Sp. Sc. 37; 425 (1982).

[12]Heller. M.,Acta. Cosm. Z11; 11 ( 1982).

[13]Herrera. L., Jimenez, J. and Ruggeri, G.J. Phys. Rev. D22; 2305 ( 1980).

[14]Krori.K.D. and Paul, B.B., J. Math. Phys. 23; 126 ( 1982).

[15]Lyttleton R.A., and Bondi. H., Proc. Roy. Soc. London. A252; 313 (1959).

[16] Mac Callum M.A.H., Gen. Rel. An Einstein Centenary Survey S.W.H. Hawking and Israel els; Cambridge University Press, Cambridge.

[17] Maniharsingh Kj., Astrophys. Sp. Sc. 148;149 (1088)

[18] Maniharsingh Kj., Astrophy. Sp. Sc. 162; 129 (1989)

[19] Maniharsingh Kj. Astrophy. Sp. Sc. 172; 29 (1990).

[20]Maniharsingh Kj., Astrophy. Sp. Sc. 180; 253 (1991).

[21] Maniharsingh Kj. Astrophy. Sp. Sc. 202; 247 (1993).

[22] Maniharsingh Kj., Gen. Rel. Grav. 27; 1145 (1995).

[23] Maniharsingh Kj. And Jugeshwor N. Can. J. Phys. (in press). N.Jugeshwor Singh. IJMSEA Vol.8 No. I 293(2014)

[24]Prakash S., Aeta Phys. Polon. B12; 3 (1981).

[25]Reddy D.R.K. and Rao V.O.M. Ind. J. Pure Appl. Phys. 20; 325 (1982).

[26]Roy S.R. and Tiwari O.P. , Ind. J. Phys. 54B ; 548 (1980).

[27] Roy S.R. and Singh J.P., Astroph. Sp. Sc. 96; 303 (1983).

[28] Singh R.T., Ph. D. Thesis I.I.T. Kharagpur, India (1980) 